\title{
Need for Vitreous Surgeries in Proliferative Diabetic Retinopathy in 10-Year Follow-Up: India Retinal Disease Study Group Report No. 2
}

\author{
Rehana Khan ${ }^{a}$ Janani Surya ${ }^{a}$ Ramachandran Rajalakshmi ${ }^{\text {b }}$ \\ Padmaja Kumari Rani ${ }^{c}$ Giridhar Anantharaman ${ }^{d}$ Mahesh Gopalakrishnan ${ }^{d}$ \\ Alok Sen ${ }^{\mathrm{e}}$ Abhishek Desai ${ }^{\mathrm{f}}$ Rupak Roy ${ }^{\mathrm{a}}$ Sundaram Natarajan ${ }^{9}$ \\ Lanin Chen ${ }^{g}$ Gajendra Chawla ${ }^{\mathrm{h}} \quad$ Umesh Chandra Behera ${ }^{\mathrm{i}}$ Lingam Gopal ${ }^{j}$ \\ Vinata Muralidharan ${ }^{a}$ Sobha Sivaprasad ${ }^{k}$ Rajiv Raman ${ }^{a}$
}

aShri Bhagwan Mahavir Vitreoretinal Services, Sankara Nethralaya, Chennai, India; 'bepartment of Ophthalmology, Madras Diabetes Research Foundation \& Dr. Mohan's Diabetes Specialities Centre, Chennai, India; 'Smt Kanuri Santhamma Centre for Vitreoretinal Diseases, LV Prasad Eye Institute, Hyderabad, India; ${ }^{\mathrm{d} D e p a r t m e n t}$ of Vitreo-Retina, Giridhar Eye Institute, Kochi, India; 'Department of Retina and Uvea, Sadguru Netra Chikitsalaya, Chitrakoot, India; ' Department of Vitreo Retinal Services, Shri Ganapati Nethralaya, Jalna, India; ${ }^{9}$ Department of Vitreo Retina Surgery, Aditya Jyot Eye Hospital, Mumbai, India; 'Director, Vision Care \& Research Centre, Bhopal, India; 'Department of Retina and Vitreous Service, LV Prasad Eye Institute, Bhubaneswar, India; ${ }^{\text {Asssociate }}$ Professor, Senior consultant, National University of Singapore, Singapore, Singapore; ${ }^{\mathrm{N}} \mathrm{NIHR}$ Moorfields Biomedical Research Centre, London, UK

\section{Keywords}

Proliferative diabetic retinopathy $\cdot$ Pan retinal photocoagulation - Anti-vascular endothelial growth factor · Vitrectomy

\begin{abstract}
Introduction: To report the 10-year rate of vitrectomies and the associated factors in people with proliferative diabetic retinopathy (PDR) from a multicentric cohort of people with diabetes mellitus. Methods: Ten centres in India with established vitreoretinal (VR) services for over 10 years were invited to provide long-term data on PDR. People with Type 1 or 2 diabetes with a clinical diagnosis of active PDR in 1 or both eyes were included. Baseline data collected included
\end{abstract}

karger@karger.com www.karger.com/ore

Karger" age, sex, duration of diabetes, source of referral and bestcorrected visual acuity, and diabetic retinopathy status in both eyes. Available follow-up data included the numbers of panretinal photocoagulation (PRP) sessions, cataract surgery, treatment of diabetic macular oedema, use of anti-vascular endothelial growth factor (VEGF) therapy, vitrectomy with or without retinal surgeries over 10 years. Results: Over 10 years, 89\% needed supplemental PRP after initial complete PRP. One-third required retinal surgery, 16\% needed intravitreal injection. Men (74.5\%) had significant higher risk for vitreous (VR) surgery. Of the group with low-risk PDR, $56.8 \%$ did not require VR surgery, $p<0.001$. Of the patients who underwent cataract surgery and had intravitreal antiVEGF injections, 78.5 and $28.2 \%$ needed subsequent vitreous (VR) surgery, $p=0.006$ and $<0.0001$, respectively. Inde- 
pendent predictors of need for vitreoretinal surgery included those who underwent cataract surgery and those with poor baseline visual acuity (logMAR). Eyes at lower risk for VR surgery included the eyes previously treated with PRP and low-risk PDR at baseline. Conclusion: Despite initial "complete" PRP, one-third of our study cohort needed vitrectomies over 10 years, highlighting that these patients require regular follow-up for a long period of time.

(c) 2020 S. Karger AG, Basel

\section{Introduction}

Proliferative diabetic retinopathy (PDR) is a leading cause of severe visual impairment in people with diabetes [1]. Several randomized clinical trials have evaluated the efficacy of panretinal photocoagulation (PRP) in patients with PDR [2-6]. The Diabetic Retinopathy Study (DRS) showed that PRP reduced the risk of severe vision loss (defined as visual acuity of 20/800 or worse at 2 consecutive 4-month visits) caused by complications of PDR from 14.0 to $6.2 \%$ during a 2 -year period and from 33.0 to $13.9 \%$ during a 5 -year period [7]. In addition, early PRP reduces the need for vitrectomy. In the Early Treatment for Diabetic Retinopathy Study (ETDRS), the 5-year vitrectomy rate was $2.1 \%$ in the group assigned to early full scatter photocoagulation as the initial treatment, $2.5 \%$ in eyes assigned to early mild scatter photocoagulation, and $4.0 \%$ in the deferred treatment group [8]. Recent clinical trials have also provided evidence that anti-vascular endothelial growth factors (anti-VEGFs) are superior to PRP for PDR in the short-term. Anti-VEGF therapies are now the standard of care for patients with diabetic macular oedema (DME), and as DME coexists with PDR, anti-VEGF is also now used in managing PDR at least in the initial stages or in combination with PRP. Diabetic Retinopathy Clinical Research (DRCR) study [9] suggests that anti-VEGF is more effective for visual outcomes than PRP and has a lesser chance of developing DME or undergoing vitrectomy and also had less visual field loss. Similarly, CLARITY study group [10] showed that patients treated with intravitreal aflibercept for PDR had a better outcome than those treated with standard PRP at 52 weeks.

Despite effective treatment options for PDR, treatment delay and inertia to complete PRP adequately remain a major challenge in the management of PDR. The diabetic retinopathy vitrectomy study [11] indicated favourable outcome with early vitrectomy over deferral (vision $10 / 20$ or better). There is a lack of long-term data from India in terms of outcomes of PDR management and the associated risk factors for need for vitrectomies. There is also an unmet need to address the knowledge gap on the practice patterns in the management of PDR and its complications in India.

In this study, we aimed to study the practice patterns of the management of PDR over the last 10 years across 10 institutions with dedicated vitreoretinal (VR) units that cater to a high throughput of patients with PDR. In particular, we evaluated the factors that were associated with the need for vitrectomy.

\section{Methods}

This study protocol was approved by the Institutional Review Board (Ethics Committee) at Vision Research Foundation, Chennai. The ten centres (tertiary care hospitals) in India with established (VR) services were chosen based on the availability of 10year data for PDR patients (Fig. 1). As it is a retrospective study, informed consent for this study was waived by the Ethics Committee and it adhered to the tenets of the Declaration of Helsinki.

Patients with a diagnosis of type 1 or 2 diabetes with active PDR in 1 or both eyes, who presented to these centres and had longterm follow-up for up to 10 years after treatment were included in this study. The study population consisted of treatment-naive PDR and as well as those with persistent PDR post-initial PRP. The patient data were identified either from electronic patient records or manually from registers maintained since 2008 to allow for outcome measurements at 10 years. The research team prepared an excel sheet and shared it with the centres to collect anonymized retrospective data from consecutive patients who met the inclusion criteria. Though the distribution of cases is across the country, it does not represent the diversities of the population of India, as the sample is drawn from eye hospitals in select urban and semiurban areas.

Baseline data collected included age, gender, duration of diabetes, source of referral and best-corrected visual acuity, diabetic retinopathy status in both eyes, presence of DME, and history of previous sessions of PRP. Low-risk PDR was defined as presence of retinal neovascularization elsewhere or disc neovascularization that did not meet high-risk characteristics. High-risk PDR was defined according to the DRS study [12] as presence of at least 3 highrisk characteristics that included presence of vitreous haemorrhage $(\mathrm{VH})$ or pre-retinal haemorrhage with the presence of any active neovascularization, location of neovascularization on or within 1 disc diameter of the optic disc or disc neovascularization $>1 / 3$ disc area or neovascularization elsewhere $>1 / 2$ disc area. Presence of $\mathrm{VH}$, retinal detachment, fibrovascular proliferation, and neovascular glaucoma was also recorded.

Previously lasered or prior lasered eyes are the eyes which had undergone initial complete PRP. Additional PRP sessions, for PDR after the completion of PRP at the initial visit, were termed supplemental PRP. The numbers of PRP sessions, need for cataract surgery, treatment of DME, use of intravitreal injections and antiVEGF therapy, and vitrectomy with or without retinal surgeries over 10 years were collected to evaluate the long-term treatment 
Fig. 1. Study sites and the number of patients recruited from each site $(\mathrm{N})$. The image was downloaded from the publicly available database which can be used for any personal use, https://images.app.goo. gl/oPMqVzKZtv83HRSA7 (June 9, 2020). Further editing was done by the corresponding author, Dr. Rajiv Raman.

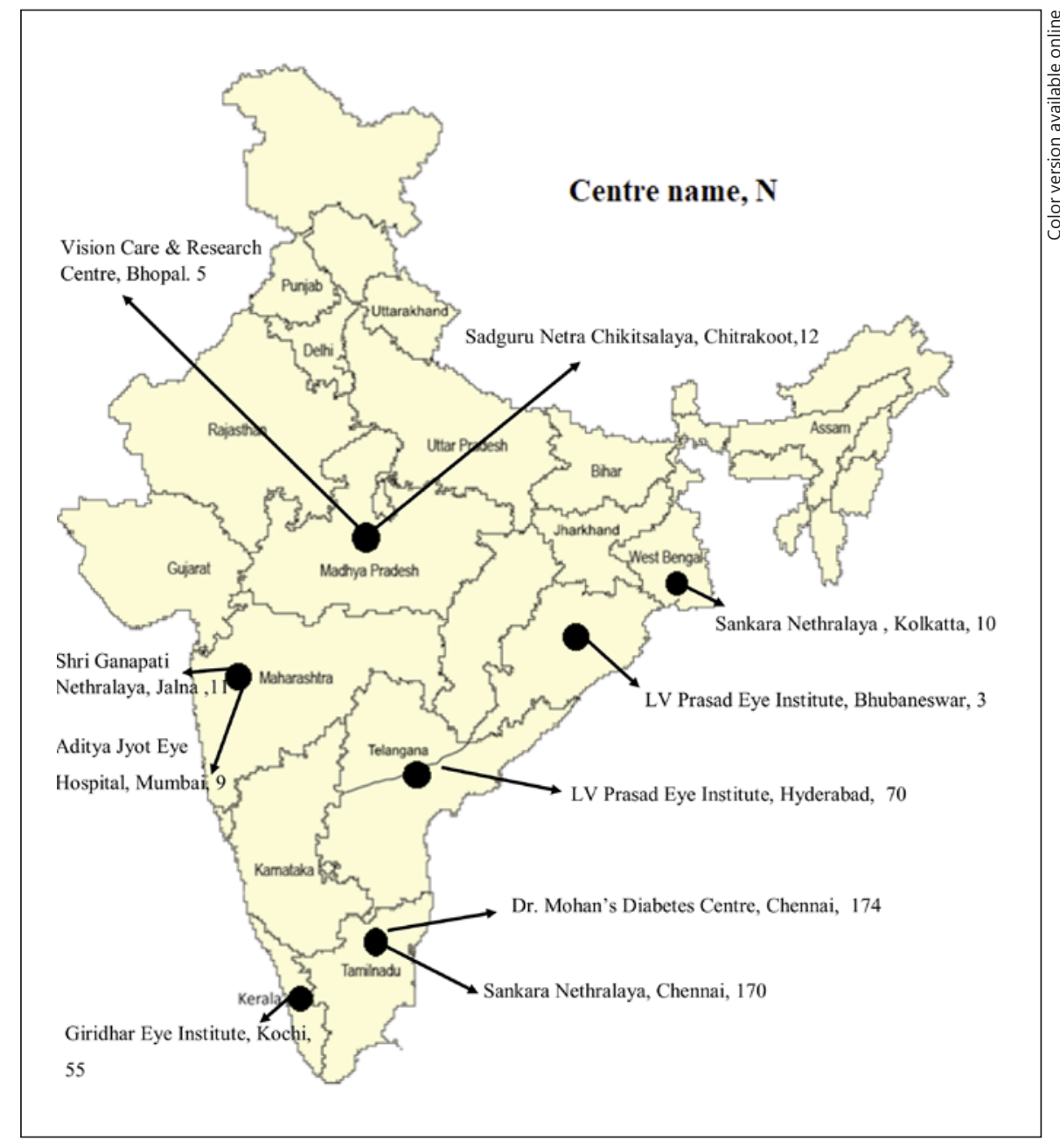

requirements for patients with PDR. The treatment choice in cases of PDR was PRP. In the event of non/partial regression of neovascularization, supplemental PRP was done. If during follow-up, there was non-clearing $\mathrm{VH}$, tractional retinal detachment involving or threatening the fovea and combined tractional and rhegmatogenous detachment. In this cohort, intravitreal anti-VEGF agents were used for centre-involving DME and in some cases prior of vitreous (VR) surgery and not as a primary treatment modality for PDR (alone or in combination with PRP).

\section{Statistical Analysis}

SPSS (version 21.0) was used for statistical analysis. Continuous data are expressed as mean \pm standard deviation, while categorical data are presented as proportions. To compare characteristics between the 2 groups, independent $t$ and $\chi^{2}$ tests were used to compare means and proportions respectively. Cox regression model was used to calculate the hazard ratio (HR) for the need of vitrectomy. Survival analysis was carried out using the KaplanMeier curve. For all statistical tests, $p$ value of $<0.05$ was considered statistically significant.

\section{Results}

A total of 1,038 eyes of 519 patients fulfilled the eligibility criteria for the study with follow-up data within the period from January 2009 to December 2019 (Fig. 1) shows the distribution of study participants recruited for the study across different geographic regions in India. The mean age of the study cohort was $52.3 \pm 9.2$ years, and mean duration of diabetes was $16.1 \pm 9.2$ years (Fig. 2) shows the 10-year change in PDR.

Table 1 shows the baseline characteristics of treatment naïve active PDR eyes versus previously lasered active PDR eyes. Over 57\% of eyes that had not undergone any treatment and $46 \%$ of those who had undergone previous laser photocoagulation therapy were low-risk PDR ( $p=$ $0.03)$. There was no significant variation in clinical presentations between treatment naïve and previously treated eyes within the high-risk PDR group. 


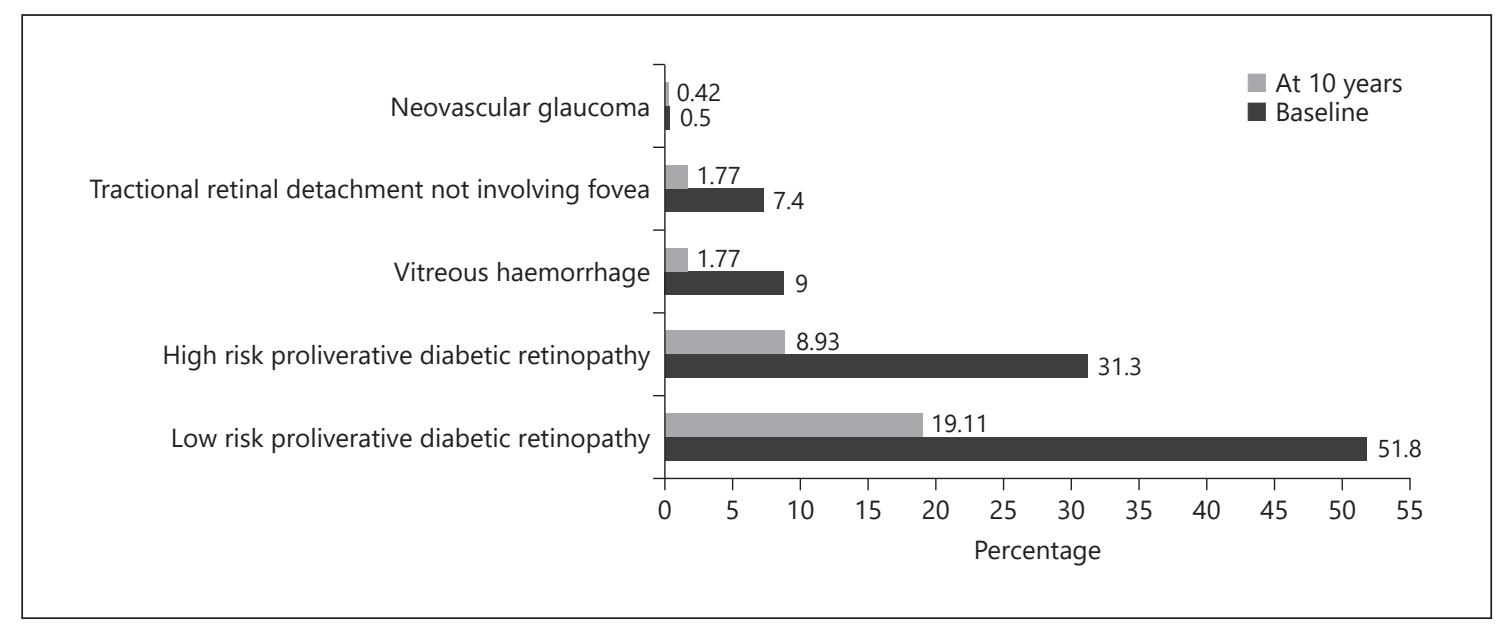

Fig. 2. Ten-year changes in PDR in the study group. PDR, proliferative diabetic retinopathy.

Table 1. Baseline characteristics of previously lasered versus non-lasered eye

\begin{tabular}{lcccc}
\hline Baseline PDR status & $\begin{array}{l}\text { Overall, } \\
n=1,038 \text { eyes }\end{array}$ & $\begin{array}{l}\text { Previously } \\
\text { lasered eyes, } \\
n \text {-460 eyes }\end{array}$ & $\begin{array}{l}\text { Treatment } \\
\text { naïve PDR, } \\
n \text {-578 eyes }\end{array}$ & $p$ value \\
\hline Age, years & $52 \pm 9$ & $53 \pm 8$ & $52 \pm 10$ & $\mathbf{0 . 0 0 1}$ \\
Gender, $n$ (\%) & $724(69.7)$ & $338(73.5)$ & $386(66.6)$ & $\mathbf{0 . 0 1 7}$ \\
$\quad$ Male & $314(30.3)$ & $122(26.5)$ & $192(33.4)$ & 0.164 \\
$\quad$ Female & $16.1 \pm 9.2$ & $16.2 \pm 8.7$ & $15.9 \pm 9.2$ & $\mathbf{0 . 0 2 8}$ \\
Duration of DM, years & $499(51.8)$ & $205(46)$ & $294(56.9)$ & 0.710 \\
High-risk PDR & $301(31.3)$ & $143(32.1)$ & $158(30.6)$ & 0.880 \\
VH & $87(9)$ & $40(9)$ & $15(9.1)$ & 0.111 \\
TRD not involving fovea & $71(7.4)$ & $56(12.6)$ & $3(0.6)$ & 0.662 \\
NVG & $5(0.5)$ & $2(0.4)$ & & \\
\hline
\end{tabular}

PDR, proliferative diabetic retinopathy; $\mathrm{VH}$, vitreous haemorrhage; TRD, tractional retinal detachment; NVG, neovascular glaucoma; DM, diabetes mellitus. Values in bold are statistically significant.

Table 2 shows the proportion of eyes that underwent interventions up to 10 years. Over the period of 10 years, $89 \%$ needed supplemental PRP, especially the previously non-lasered eyes ( 92.2 vs. $85 \%, p$ 0.004). After the initial baseline PRP (2-3 sessions), during the 10-year followup, the overall average number of further PRP sessions were 3.03 sessions, in previously lasered eyes 2.58 sessions and in treatment naïve PDR eyes 3.39 sessions $(p<$ 0.0001 ). A third of patients required retinal surgery, with no statistical difference between previously lasered and non-lasered eyes at baseline. Overall, 16\% of eyes were also treated with intravitreal anti-VEGF injections during the 10-year period, with more previously lasered eyes re- quiring this treatment option than treatment naïve eyes ( 20 vs. $12.9 \%, p=0.00$ ).

Table 3 shows the factors associated with the need for VR surgery in the study population during the 10-year follow-up. Men (74.5\%) were more likely to require VR surgery. As expected, eyes with low-risk PDR were less likely to require VR surgery. Other significant factors associated with VR surgery included poor baseline bestcorrected visual acuity, cataract surgery, and anti-VEGF therapy. In univariate analysis, risk factors for the need for VR were lower baseline visual acuity, eyes that underwent cataract surgery or anti-VEGF injection, and nonlasered eyes at baseline. After adjustment of variables in- 
Table 2. Interventions during the 10 -year follow-up in of previously lasered versus non-lasered patients

\begin{tabular}{lcccc}
\hline Interventions during 10-year follow-up & $\begin{array}{l}\text { Overall, } \\
n=1,038 \text { eyes }\end{array}$ & $\begin{array}{l}\text { Previously } \\
\text { lasered eyes, } \\
n \text {-460 eyes }\end{array}$ & $\begin{array}{l}\text { Treatment } \\
\text { naïve PDR, } \\
n-578 \text { eyes }\end{array}$ & $p$ value \\
\hline Supplemental PRP, $n(\%)$ & $924(89)$ & $391(85)$ & $533(92.2)$ & $\mathbf{0 . 0 0 4}$ \\
Average number of PRP sessions & $3.03 \pm 2.03$ & $2.58 \pm 1.79$ & $3.39 \pm 2.14$ & $<\mathbf{0 . 0 0 0 1}$ \\
Cataract surgery & $620(59.7)$ & $302(65.6)$ & $318(55)$ & 0.751 \\
VR surgery & $326(31.4)$ & $185(40.2)$ & $141(24.4)$ & 0.160 \\
Intravitreal anti-VEGF & $167(16)$ & $92(20)$ & $75(12.9)$ & $<\mathbf{0 . 0 0 0 1}$ \\
\hline
\end{tabular}

PRP, panretinal photocoagulation; VR, vitreoretinal; Anti-VEGF, anti-vascular growth factor; PDR, proliferative diabetic retinopathy. Values in bold are statistically significant.

Table 3. Risk factors for the need for VR surgery in the study population

\begin{tabular}{|c|c|c|c|}
\hline Risk factors & $\begin{array}{l}\text { Underwent } \\
\text { VR surgery, } \\
n-326 \text { eyes }\end{array}$ & $\begin{array}{l}\text { Did not need } \\
\text { VR surgery, } \\
n-637 \text { eyes }\end{array}$ & $p$ value \\
\hline Mean age, years \pm SD & $51.62 \pm 8.65$ & $52.61 \pm 9.32$ & 0.097 \\
\hline Duration of diabetes, years, mean \pm SD & $16.07 \pm 9.38$ & $15.98 \pm 8.8$ & 0.880 \\
\hline Men, $n(\%)$ & $243(74.5)$ & $478(67.4)$ & 0.053 \\
\hline Women, $n(\%)$ & $83(25.5)$ & $231(32.6)$ & 0.201 \\
\hline Low-risk PDR, $n(\%)$ & $96(29.4)$ & $403(56.8)$ & $<0.0001$ \\
\hline High-risk PDR, $n(\%)$ & $107(32.8)$ & $194(27.4)$ & 0.317 \\
\hline $\mathrm{VH}, n(\%)$ & $48(14.7)$ & $39(5.5)$ & 0.167 \\
\hline TRD not involving fovea, $n(\%)$ & $55(16.9)$ & $16(2.3)$ & 0.136 \\
\hline NVG, $n(\%)$ & $2(0.61)$ & $3(0.42)$ & 0.920 \\
\hline Underwent cataract surgery, $n(\%)$ & $256(78.5)$ & $364(51.3)$ & $<0.0001$ \\
\hline Underwent intravitreal anti-VEGF injection, $n(\%)$ & $92(28.2)$ & $75(10.6)$ & 0.006 \\
\hline Previously not lasered, $n(\%)$ & $48(14.7)$ & $63(8.9)$ & $<0.0001$ \\
\hline Previously lasered, $n(\%)$ & $185(56.75)$ & $276(43.32)$ & $<0.0001$ \\
\hline Baseline visual acuity (LogMAR), mean \pm SD & $0.79 \pm 0.89$ & $0.35 \pm 0.54$ & $<0.0001$ \\
\hline
\end{tabular}

VR, vitreoretinal; PDR, proliferative diabetic retinopathy; VH, vitreous haemorrhage; $T R D$, tractional retinal detachment; NVG, neovascular glaucoma; VEGF, vascular endothelial growth factor; SD, standard deviation. Values in bold are statistically significant.

cluding the age of diagnosis of PDR (years), gender (male), duration of diabetes (years) and mean baseline visual acuity (log MAR) in the multivariate model, variables that were independent predictors of need for VR surgery included those who underwent cataract surgery (HR: $2.84,95 \%$ CI 2.16-3.74; $p<0.001$ ) and those with poor presenting visual acuity (Log MAR) at baseline (HR: $1.31,95 \%$ CI 1.19-1.44; $p<0.001$ ). The eyes at lower risk for VR surgery included the eyes previously treated with PRP (HR: 0.72, 95\% CI 0.53-0.99; $p=0.04$ ) and low-risk PDR at baseline (HR: 0.47, 95\% CI 0.36-0.60; $p<0.001)$.

\section{Discussion}

This collaborative study on a PDR cohort from India with 10-year follow-up shows the practice patterns for management of PDR in India in people who are under continual care in the respective institutions with vitreoretinal services. This study population represent subjects from heterogeneous socioeconomic strata symbolic of Indian population.

This study has highlighted some important points. Firstly, this study shows that PRP is the mainstay of treatment for PDR in India. The mean age of patients with 
PDR was approximately 55 years, and they were predominantly males with mean duration of diabetes was about 15 years. These demographics are similar to those of recent randomized controlled studies done in the high-income countries $[9,10]$. However, $>40 \%$ of patients presented with high-risk PDR features, including 9\% with $\mathrm{VH}$ and 3\% with neovascular glaucoma. This highlights the lack of systematic screening for sight-threatening DR. In contrast, in the CLARITY study [10] done on PDR in the United Kingdom, it was challenging to recruit patients with high-risk PDR $(80 \%$ of the cohort recruited were low-risk PDR). This difference in presenting features of PDR emphasizes the need for systematic screening for DR in India. We have previously reported the prevalence and incidence of visual impairment in PDR in India based on this study cohort [13]. We found that the outcome of visual acuity was better in eyes receiving treatment at early stage than deferred to a later stage. Stable treated PDR eyes maintained visual acuity, showing that inadequate treatment in the persistent disease activity group resulted in worse visual outcomes over 10 years.

Another interesting result noted was that the clinical features of treatment naïve PDR patients were similar to previously treated PDR patients, suggesting that repeated PRP sessions may not be applied in the initial stages to control the disease. Although initial undertreatment with PRP is an assumption and cannot be verified in this study, another point in this study that substantiates the difficulty in controlling the disease in India is that $89 \%$ of the cohort required supplemental therapy over 10 years. Our data showed that the previously lasered eyes had inadequate PRP sessions, as we found that the previously lasered eyes required supplemental PRP sessions during the 10-year follow-up suggesting that the prior laser provided at baseline was incomplete. In the clinical trials on PRP [14], 27 and 11\% required supplemental therapy by 2 and 5 years, respectively.

With the advent of anti-VEGF therapy for the treatment of central-involved DME (CI-DME), it has been recognized that intravitreal anti-VEGF therapy reduced the severity of DR and the risk of worsening to increased severity of DR [15]. In 2-year outcome, results of the DRCR Protocol S, which compared the safety and efficacy of PRP (203 eyes) to intravitreal injection ranibizumab $0.5 \mathrm{mg}$ injections (191 eyes) for the treatment of PDR showed that ranibizumab was non-inferior to PRP for treatment of PDR with a mean gain of 2.8 letters [9]. Approximately, $16 \%$ of this patient cohort received antiVEGF, although the indication (DME or before vitrectomy) for this treatment option and type of anti-VEGF were not collected. In India, the usage of anti-VEGF for DME has increased widely over the last few years. Its usage in PDR is limited. However, as patients incur the cost of these drugs as out of pocket expenses, its usage is erratic. More eyes in the previously treated PRP group received anti-VEGF compared to treatment naïve group. Secondary outcomes suggest that other aspects of visual functions may be better preserved with anti-VEGF than with PRP treatment. A study by Irini et al. [16] showed that there were no changes in the visual acuity or retinal thickness noted when anti-VEGF was used alone or in combination with PRP for the treatment of PDR.

A third of patients required VR surgery by 10 years, irrespective of the PRP status at baseline, again highlighting the disease severity of the patients in this cohort. In the DRVS study [11] done in 1985, vitrectomy was undertaken only if the retinal detachment involved the centre of the macula or for non-clearing $\mathrm{VH}$ after a 1-year waiting period, and $25 \%$ of eyes required vitrectomy after 2 years of follow-up. In the ETDRS study where vitrectomy was mainly done for $\mathrm{VH}$ or retinal detachment, the 5-year vitrectomy rates were $5.6 \%$ [8]. In more recent studies, $\mathrm{VH}$ which required vitrectomy was observed in only $6 \%$ at 1 year in the CLARITY study [10] only 15 and $29 \%$ required vitrectomy at 2 and 5 years in Protocol S $[9,14]$. Our study results are similar to the DRVS study, reiterating undertreatment during initial PRP in this study cohort. However, as our study is retrospective and all other studies are randomized control trials, the results cannot be directly compared. In the study done by Parikh et al. [17], in 374 eyes of 272 patients after initial PRP, PDR with associated $\mathrm{VH}$ or traction were more likely to undergo vitrectomy within 2 years following initial PRP compared with eyes with only PDR. Irini et al. [18], has shown for the patients who had been treated with pars plana vitrectomy, intravitreal ranibizumab seems to be an effective treatment in patients with recurrent $\mathrm{VH}$. In our study, these risk factors, $\mathrm{VH}$, and traction at baseline were seen in 9 and $7.4 \%$, respectively. These cases probably also contributed to those with poor vision at baseline. We also observed cataract surgery (HR 2.84, $p<0.001$ ) and poor presenting vision as risk factors for VR surgery (HR 1.31, $p<0.001$ ). Approximately, $60 \%$ required cataract surgery in our cohort. Similar to our study, Ruiz et al. [19] reported in their series that $20 \%$ of the patients with inactive lasered PDR progressed after cataract surgery.

The main strengths of this study are the large sample of 1,038 eyes of 519 patients with PDR who has been followed up for 10 years across 10 retinal departments across India. This study has underscored various ways by which 
PDR care can be improved in India. Firstly of all, PRP is the first-line treatment and should continue to do so. However, a systematic screening programme for DR is required urgently to ensure that patients present with less severe disease. Secondly, PRP should be completed as early and effectively as possible to achieve disease stability. Therefore, patients need to be educated for the need for urgent and regular frequent PRP sessions until the PDR stabilises. If anti-VEGF therapy is given for DME, concomitant PRP should be given to control the PDR. Cataract surgery is associated with increased risk for VR surgery, and so PDR should be optimally treated prior to the surgery or orienting cataract surgeons and general ophthalmologists about the risk of progression of PDR following cataract surgery is important. This awareness creation will also help to treat these eyes adequately following cataract surgery.

Absence of parameters on systemic conditions such as glycaemic control, blood pressure, nephropathy, and dyslipidemia is a major limitation of this study. Due to the inherent limitation of retrospective study, there was lack of standardization of the phenotyping and visual acuity assessments. These findings are based on PDR data of Retina clinics in India and cannot be extrapolated to the population.

This study is based on patients with long-term followup data. In real life, this group of patients are at high risk of other complications of diabetes and mortality. They fail to attend clinic appointments even in the most developed health systems, and so the outcomes of PDR patients are likely to be worse than these results. In Protocol S, only $66 \%$ of the patients (excluding mortality) completed the study. However, the outcomes of this multicentre study done across India serves as a benchmark for management of PDR patients in India.

In conclusion, we found that over 10-year follow-up, despite PRP, one-third of our study cohort needed VR surgery. There is an urgent need for systematic DR screening programs to identify PDR in early stages as low-risk PDR. It re-emphasizes the fact that regular follow-up at repeated interval and appropriate treatment can reduce the vision loss in patients with PDR.

\section{Acknowledgement/Funding Sources}

This work was supported by the Global Challenges Research Fund and UK Research and Innovation through the Medical Research Council grant number MR/P027881/1 and the National Institute for Health Research (NIHR) Biomedical Research Centre based at Moorfields Eye Hospital NHS Foundation Trust and UCL Institute of Ophthalmology.

\section{Statement of Ethics}

This study protocol was approved by the Institutional Review Board (Ethics Committee) at Vision Research Foundation, New No 41, Old No 18, College Road, Chennai-600006 (Study reference number: 674A-2018-P), Approval Date - March 22, 2018. Informed consent for this study was waived by the Vision Research Foundation Ethics Committee, which is working according to ICH-GCP and adheres to the tenets of the Declaration of Helsinki.

\section{Conflict of Interest Statement}

The authors have no conflicts of interest to declare.

\section{Author Contributions}

R.R. ${ }^{1(\#)}$, S.S., and R.R. ${ }^{(2)}$ contributed in conception and design of the study. R.R. ${ }^{1(\#)}$. and R.K. wrote the main manuscript text and prepared all the tables. R.K. and J.S. assisted with statistical analyses. S.S., R.R. (2)., G.A., U.C.B., and P.K.R. drafted the work and substantively revised it. R.R. ${ }^{1(*)}$.,P.K.R., A.S., A.D., S.N., L.C., G.C., M.G., U.C.B., and V.M. helped in acquisition of the data. All authors reviewed the manuscript.

\section{References}

1 Zheng Y, He M, Congdon N. The worldwide epidemic of diabetic retinopathy. Indian $\mathrm{J}$ Ophthalmol. 2012 Sep;60(5):428.

2 Klein R, Klein BEK, Moss SE. Visual impairment in diabetes. Ophthalmology. 1984;91: $1-9$.

3 The Diabetes Control and Complications Trial Research Group, Nathan DM, Genuth S, Lachin J, Cleary P, Crofford O. The effect of intensive treatment of diabetes on the development and progression of long-term complications in insulin-dependent diabetes mellitus. N Engl J Med. 1993;329:977-86.
4 The Diabetic Retinopathy Study Research Group. Photocoagulation treatment of proliferative diabetic retinopathy. Clinical application of Diabetic Retinopathy Study (DRS) findings, DRS report number 8 . Ophthalmology. 1981;88:583-600.

5 Early Treatment Diabetic Retinopathy Study Research Group. Photocoagulation for diabetic macular edema: Early Treatment Diabetic Retinopathy Study report number 1 . Arch Ophthalmol. 1985;103:1796-806.

6 Early Treatment Diabetic Retinopathy Study Research Group. Early photocoagulation for diabetic retinopathy: ETDRS report number 9. Ophthalmology. 1991;98(5 Suppl 1):76785.

7 Michael WS. Diabetic Retinopathy: Current Pharmacologic Treatment and Emerging Strategies. Singapore: Springer Verlag; 2018.

8 Flynn HW Jr, Chew EY, Simons BD, Barton FB, Remaley NA, Ferris FL III. Early treatment diabetic retinopathy study research group. Pars plana vitrectomy in the early treatment diabetic retinopathy study: ETDRS report number 17. Ophthalmology. 1992 Sep 1;99(9):1351-7. 
9 Gross JG, Glassman AR, Jampol LM, Inusah S, Aiello LP, Antoszyk AN, et al. Panretinal photocoagulation vs intravitreous ranibizumab for proliferative diabetic retinopathy: a randomized clinical trial. Jama. $2015 \mathrm{Nov}$ 24;314(20):2137-46.

10 Sivaprasad S, Prevost AT, Vasconcelos JC, Riddell A, Murphy C, Kelly J, et al. Clinical efficacy of intravitreal aflibercept versus panretinal photocoagulation for best corrected visual acuity in patients with proliferative diabetic retinopathy at 52 weeks (CLARITY): a multicentre, single-blinded, randomised, controlled, phase $2 \mathrm{~b}$, non-inferiority trial. The Lancet. 2017 Jun 3;389(10085):2193-203.

11 The Diabetic Retinopathy Vitrectomy Study Research Group. Early vitrectomy for severe vitreous hemorrhage in diabetic retinopathy. Two-year results of a randomized trial. Diabetic retinopathy vitrectomy study report 2 . Arch Ophthalmol. 1985;103(11):1644-52.

12 Diabetic retinopathy study. Report Number 6. Design, methods, and baseline results. Re- port Number 7. A modification of the Airlie House classification of diabetic retinopathy. Prepared by the Diabetic Retinopathy. Invest Ophthalmol Vis Sci. 1981;21(1 Pt 2):1-226.

13 Khan R, Chandra S, Rajalakshmi R, Rani PK, Anantharaman G, Sen A, et al. Prevalence and incidence of visual impairment in patients with proliferative diabetic retinopathy in India. Scientific Reports. 2020 Jun 29; 10(1):1-8.

14 Gross JG, Glassman AR, Liu D, Sun JK, Antoszyk AN, Baker CW, et al. Five-year outcomes of panretinal photocoagulation vs intravitreous ranibizumab for proliferative diabetic retinopathy: a randomized clinical trial. JAMA ophthalmol. 2018 Oct 1;136(10): 1138-48.

15 Gross JG, Glassman AR. A novel treatment for proliferative diabetic retinopathy: antivascular endothelial growth factor therapy. JAMA Ophthalmol. 2016;134(1):13-4.

16 Chatziralli I, Dimitriou E, Theodossiadis G, Kazantzis D, Theodossiadis P. Intravitreal ra- nibizumab alone or in combination with panretinal photocoagulation for the treatment of proliferative diabetic retinopathy with coexistent macular edema: long-term outcomes of a prospective study. Acta Diabetol. 2020 Oct; 57(10):1219-122

17 Parikh R, Shah RJ, VanHouten JP, Cherney EF. Ocular findings at initial pan retinal photocoagulation for proliferative diabetic retinopathy predict the need for future pars plana vitrectomy. Retina. 2014 Oct 1;34(10):19972002.

18 Chatziralli I, Dimitriou E, Theodossiadis G, Bourouki E, Bagli E, Kitsos G, et al. Intravitreal ranibizumab versus vitrectomy for recurrent vitreous haemorrhage after pars plana vitrectomy for proliferative diabetic retinopathy: a prospective study. Int Ophthalmol. 2020 Apr;40(4):841-7.

19 Ruiz RS, Saatci OA. Posterior chamber intraocular lens implantation in eyes with inactive and active proliferative diabetic retinopathy. Am J Ophthalmol. 1991 Feb 1;111(2):158-62. 